\title{
Influence of the Realistic Artery Geometry Parameters on a Coronary Stent Fatigue Life
}

\author{
Xinyang Cui*, ${ }^{*}$, Qingshuai Ren ${ }^{*, \dagger, \S}$ Gaoyang $\mathrm{Li}^{*}$,, Zihao $\mathrm{Li}^{*}, \|$ \\ and Aike Qiao**** \\ ${ }^{*}$ College of Life Science and Bioengineering \\ Beijing University of Technology No.100, Pingleyuan \\ Chaoyang District, Beijing, P. R. China \\ ${ }^{\dagger}$ Institute of Mechanics, Chinese Academy of Science \\ No.15, BeiSiHuanWest Road, Beijing, P. R. China \\ ${ }^{\ddagger}$ xinyangcui@qq.com \\ §Qingshuairen@qq.com \\ 『gaoyangli@qq.com \\ $\|_{z i h a o l i @ q q . c o m}$ \\ **qak@bjut.edu.cn
}

Received 4 September 2017

Accepted 10 December 2017

Published 23 January 2018

\begin{abstract}
The stents' adaptability and safety in realistic and idealized stenotic coronary model were compared to investigate the influence of artery geometry parameter on stent fatigue life. The stents' fatigue resistance ability was calculated using Goodman diagram, and the cycle to failure, the fatigue life, and the fatigue safety factor (FSF) were analyzed. Although the peak top of the von Mises stress was located at the bending area of crowns, the stress distributions were different in the two models. Considering the safety and accuracy, it is necessary to use a realistic geometric model to calculate the stent fatigue performance.
\end{abstract}

Keywords: Biomechanics; artery geometry parameters; fatigue life prediction.

\section{Introduction}

Nowadays, stent intervention has become a common method for clinical treatment of cardiovascular diseases [Lloydiones et al. (2010)]. Currently, more than $85 \%$ of coronary interventions involve stents Almangour et al. (2013)]. However, stent failure and fracture (SF) is an inherent risk of stenting Almangour et al. (2013)]. Fractured stents may lose their ability to scaffold occluded arteries and then cause restenosis Scheinert et al. (2005)], thrombosis [Bessias et al. (2005)], or artery perforation [Lewitton and Babaev (2008)]. The FDA requires that the stents should survive at least for $4 \times 10^{8}$ cycles (10 years equivalent) without exhibiting failure.

\footnotetext{
** Corresponding author.
} 
Computational models have been widely used for their ability to replicate the biomechanical response of medical devices under physiological conditions. Researchers usually simulate the stenting by using the idealized models. From a mechanical point of view, the complex biomechanical environment caused by plaques may lead to SF Morlacchi et al. (2014)]. Therefore, researchers have used realistic models in recent years to evaluate the mechanical performance of stents Auricchio et al. (2011); Ragkousis et al. (2104); Gijsen et al. (2008); Petrini et al. (2016)]. The researchers have also analyzed the fatigue parameter based on the expansion of stents by using the simple idealized models [Hsiao et al. $(2012,2014)]$. There are only a few researches on the fatigue parameter with a realistic coronary model, which provide new insights into the design and failure mechanism of the coronary stent Marrey et al. (2006)]. Dordoni et al. (2014) thought the plaque shape played an important role in the fatigue behavior. Based on these situations, the mechanical parameters and fatigue prediction by using a realistic geometry model is worth analyzing.

In this study, the mechanical comparison between an idealized model and a realistic model was performed to investigate the influence of artery geometry parameter on the fatigue life of the stent. Structural finite element models were implemented to simulate the stent expansion in a realistic model of stenotic coronary artery. The von Mises stress in the stent, plaque and vessel wall in an idealized model and a realistic model were compared to quantify the response with the presence of a realistic geometrical plaque and arterial vessel.

\section{Methods}

The mechanical loading, either monotonic or cyclic loading can cause stent failure during deployment or service, respectively. Cyclic loading is due to the pulsatile blood pressure or due to bending, torsion, or tension/compression of vessel movements to the stent Auricchio et al. (2015)]. Most of the researchers considered that the pulsatile blood pressure and vessel movement bending could affect the stent fatigue [Morlacchi et al. (2014)]. In this study, we consider that cardiac wall movement is more significant in the deformation than in the displacement, and we will focus on the fatigue failure due to pulsatile blood pressure loading to save the computational workload.

To investigate the effects of cyclic blood pressure on the fatigue resistance of the stent, the Goodman diagram as well as the fatigue performance parameters, such as the cycle to failure, the fatigue life and fatigue safety factor (FSF), were analyzed.

\subsection{Reconstruction of the $3 D$ model}

We processed the CT angiography (CTA) image of a stenotic coronary artery of a 56-year-old male patient by using Mimics 15.0. According to the CTA data of left coronary artery (LCA) lesion (Fig. T(a)), we reconstructed a 3D model of coronary vascular tree using the software "Freeform Plus 2013", as shown in Fig. T(b). 


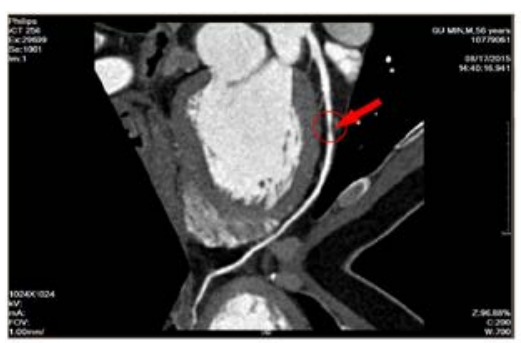

(a)

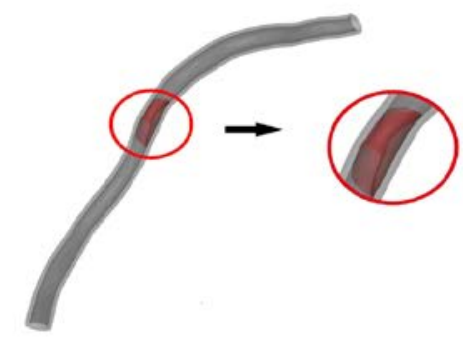

(c)

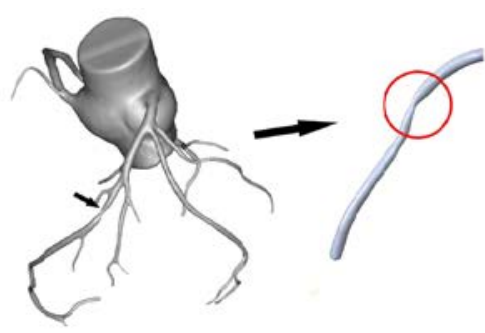

(b)

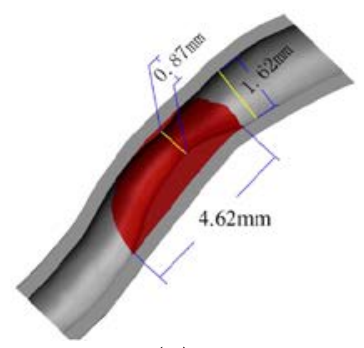

(d)

Fig. 1. (Color online) 3D model reconstruction of coronary artery. (a) CTA image of a stenosed coronary; (b) 3D reconstruction of coronary tree from CTA; (c) coronary artery lumen (dark grey), reconstructed plaque (dark red) and outer vessel wall profile (light grey); (d) the geometry size of the realistic atherosclerotic lesions of blood vessels.

The stenotic segment in the red marker illustrated the presence of the plaque. The realistic plaque geometry can be obtained from the logic subtraction between the imagined healthy artery and the stenotic artery lumen. Since the vessel wall thickness can't be obtained from the CTA images, we constructed the lumen surface by expanding $0.35 \mathrm{~mm}$ outward to establish the vessel model with uniform thickness according to the clinical data, as shown in Fig. 1(c). The internal diameter of the blood vessel was $1.62 \mathrm{~mm}$, the length of the stenosis (i.e., the length of the plaque) was $4.62 \mathrm{~mm}$, and its diameter was $0.87 \mathrm{~mm}$. The stenotic ratio of the diameter was $46.3 \%$, as shown in Fig. 1(d).

The size of the narrow blood vessels was taken into consideration to ensure the implementation of the percutaneous coronary artery. The 3D geometry of the repeatable units of the stent was generated by using Pro/Engineer Wildfire 5.0. There are six-unit cells linked by I-link bars to form a circular stent, and each single unit cell consists of 12 crowns with square shaped struts, as shown in Fig. 2]

\subsection{Meshed models and material property}

Finite Element Method (FEM) was applied to simulate the stent expansion in the coronary artery and the subsequent deformation under cyclic loading conditions for 
X. Cui et al.

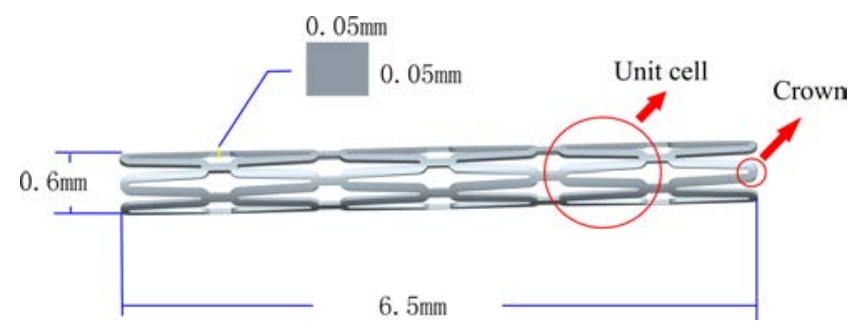

Fig. 2. Geometrical model of the stent with I-link.

diastole and systole. The commercial FEM tool, ABAQUS/Standard 6.13, was used to run the simulations.

The material properties of these models were extracted from the literature, and the stent was made of 304 stainless steel Qiao et al. (2014)]. According to Pericevic et al.'s research Pericevic et al. (2009)], the plaque without calcification or with a low level of calcification was unstable, while the stiffer calcified plaque could reduce the level of stress within the arterial tissue for a given inflation pressure. In this research, the plaque and arterial vessel wall were assumed to be calcified without rupture initially.

Software Hypermesh11.0 was used to mesh the vessel wall, plaque and the stent. Both the vessel wall and the corresponding plaque model were meshed with the tetrahedron element, which was relatively denser and would avoid the disturbance of irregular structures in a realistic stenosis model.

Since the realistic model and the stent were deformed largely in the expansion process, the stent was meshed with the linear tetrahedron element to prevent deformed interference deformation, as shown in Fig. 3(a). The stent was positioned inside the stenotic coronary artery, and the combined mesh model was shown in Fig. 3(b). The material of the vessel wall and the corresponding calcified plaque were ideally linear elastic, isotropic and incompressible, while the material of the stent was bilinear elastic-plastic. Detailed information of the combination was presented in Table 1 .

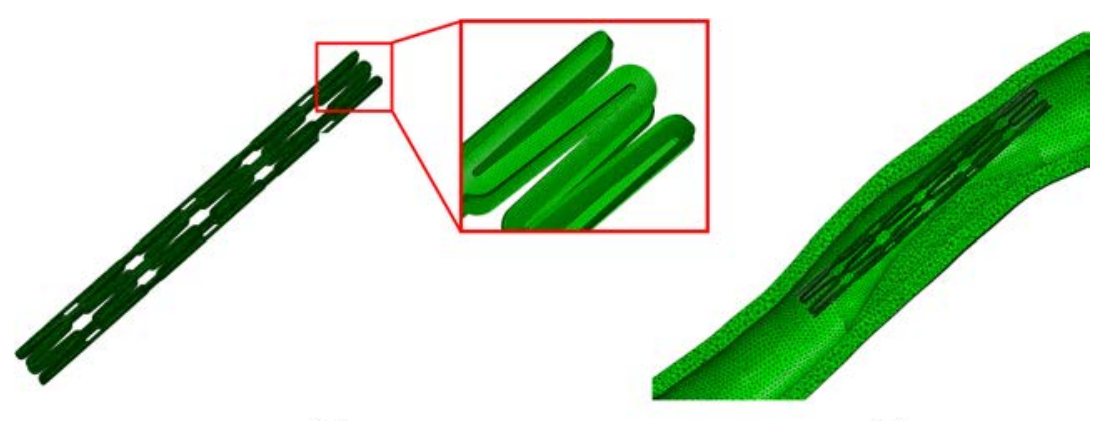

(a)

(b)

Fig. 3. Mesh of the realistic model. 
Influence of Artery Geometry on Stents Fatigue Life

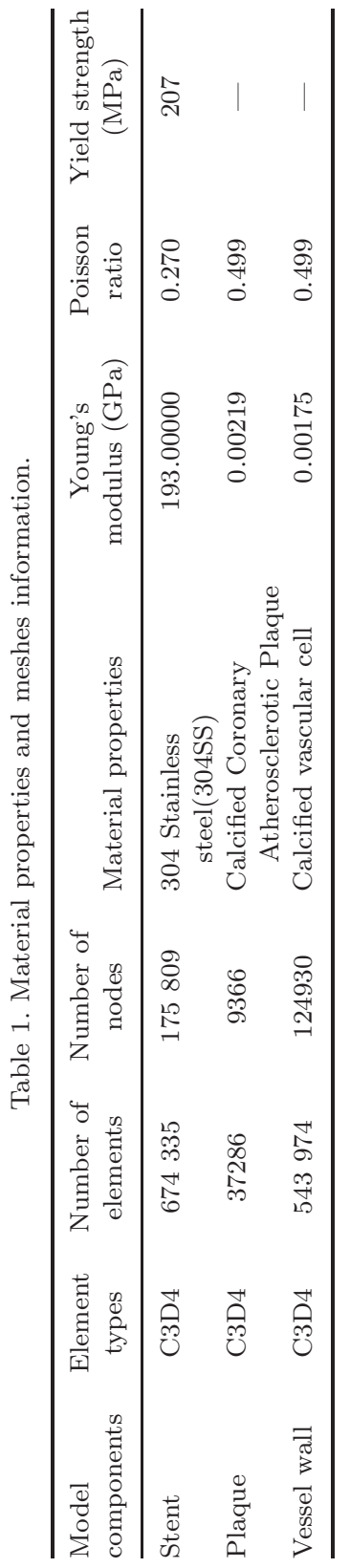




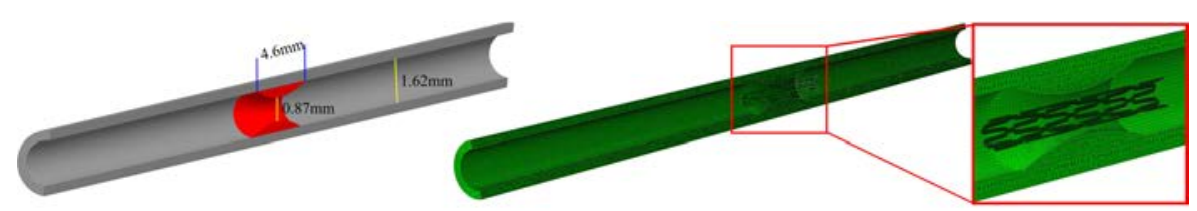

(a)

(b)

Fig. 4. Geometry (a) and mesh (b) of the idealized model.

The stent was positioned inside the coronary artery. Then, uniform radial deformation load was imposed on the stent's inner surface along the radial direction to simulate the expansion process. Furthermore, nodes belonging to the stent surface were constrained in the tangential direction to avoid any rotation inside the vessel. The artery was constrained in the section near the plaque to prevent any displacements and rotations. The friction factor of the contact surfaces between the stent and the vessel wall feature was 0.2 Yang et al. (2008)]. Plaque and the vessel wall were assumed to be bonded to ensure the models' displacement.

The simulated loading steps were as follows. First: Stent expansion. After percutaneous transluminal angioplasty (PTA), the balloon inflation was driven by displacement analysis, where a uniform radial deformation was imposed to the inner surface of the stent until the diameter is equal to 1.1 times the healthy vessel inner diameter Dordoni et al. (2014)]. At the end of this step, the deformation was deflated, and the plaque and artery vessel wall were elastically recoiled.

Second: Diastole-systole cyclic loading. After being expanded in the stenotic coronary artery, the stent underwent pulsatile loading produced from the oscillation of the internal blood pressure. The internal blood pressure oscillated between $80 \mathrm{mmHg}$ and $120 \mathrm{mmHg}$ from diastole to systole and was applied to the inner surfaces of the vessel wall. It was believed that the constitutive model response could be converged after three loading cycles Morlacchi et al. (2014)]. Diastolic heart filling and systolic contraction steps were simulated for three cardiac cycles.

\subsection{Establishment of an idealized model as the control group}

To compare the effects of realistic vascular geometry on the mechanical and fatigue properties of the stent, we established an idealized control group in which the stenotic ratio of diameter was $46.3 \%$. The geometry of the idealized model was obviously uniform and symmetric, and other information and boundary conditions were the same as the realistic coronary model. Detailed information of the idealized model is presented in Fig. 4.

\section{Results and Discussion}

\subsection{Influence of a realistic geometry: stress analysis}

The deformation loading was deflated at the end of stent expansion. The plaque and artery vessel wall were elastically recoiled, and consequently the stent protruded 

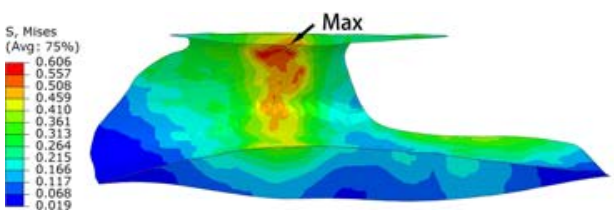

(a)
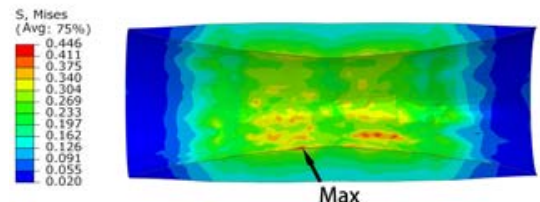

(c)
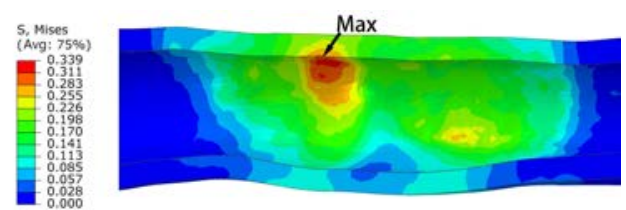

(b)
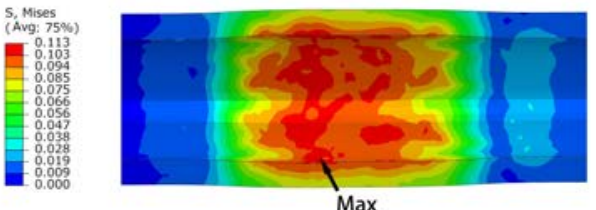

(d)

Fig. 5. Contours of the von Mises stress in the plaque and artery vessel wall (the arrow marks the location of the maximum value).

towards the center of the lumen. Figure 5 showed the contours of the von Mises stress in the stenotic artery vessel. The contours of the strain were quite similar (not shown). The higher stress region of the plaque was located in the stenosis, and there was a correlation between the stress distribution and the plaque geometric shape. The stress distributed uniformly along the axis in the idealized model (Figs. 5 (c) and 5 d)), and the distribution was different from that in the realistic model (Figs. 5 (a) and 5(b)). Quantitatively, the stenotic ratio of diameter turned to $30.8 \%$ in the realistic model, while that of the idealized model was $27.2 \%$. The peak of the von Mises stress in the plaque was $6.06 \mathrm{MPa}$ in the realistic model (Fig. 5(a)), while it was $4.46 \mathrm{MPa}$ in the idealized model (Fig. 5(c)). The relative error ratio was about $26.4 \%$ on the basis of the former one. Figures 5 (c) and 5(d) showed that the difference of stress distribution of the inner vessel wall between the realistic model and idealized model was obvious. In the higher stress region, the stress was mainly about $1.13 \mathrm{MPa}-3.39 \mathrm{MPa}$ (Fig. 5 (b)), while in the realistic model, it was mainly about $0.56 \mathrm{MPa}-1.13 \mathrm{MPa}$ (Fig. 5 (d)). The peak of the von Mises stress in the inner vessel wall was $3.39 \mathrm{MPa}$ in the realistic model, while in the idealized model, it was $1.13 \mathrm{MPa}$. The relative error ratio was about $66.7 \%$ on the basis of the former one. This revealed that the realistic geometric parameters greatly influenced the result of the biomechanics.

According to some researches [Chua et al. (2004)], the stainless stents generally have a good radial support ability of resisting radial contraction of blood vessels under pulsation to ensure that the range of the vascular radius changes was less than $0.25 \%$. Based on the above-mentioned situations, the fatigue behavior of stainless steel was stress-controlled, and the stress contours in different stages (i) stent expansion; (ii) systolic [120 mmHg]; (iii) diastolic cyclic loading [80 $\mathrm{mmHg}$ ] are depicted in Fig. 6] After the stent expansion, the plaque and the artery vessel wall were elastically recoiled. In this step, the high stress region was located in the stent struts close to the calcified plaque. The peak of the von Mises stress was $413.8 \mathrm{MPa}$ and 


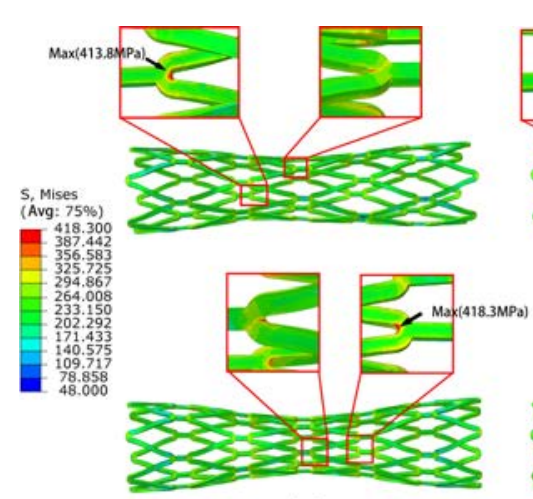

(a)

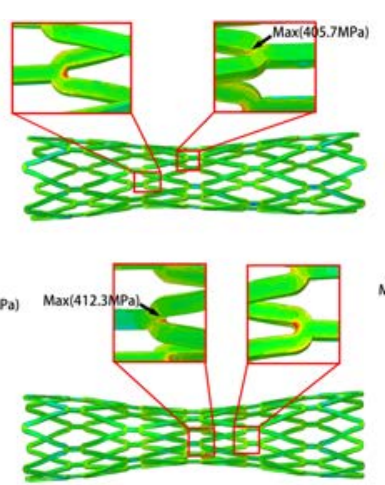

(b)

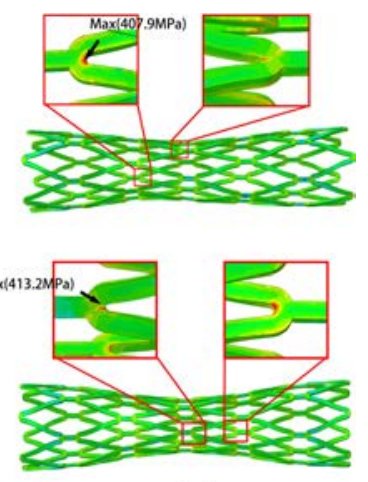

(c)

Fig. 6. Comparisons of von Mises stress in the stent under different conditions. Upper: realistic model; Lower: idealized model. (a) After recoiling; (b) Systole; (c) Diastole.

was located at the bend of the crown (Fig.6(a)). The low stress region was located in the middle position of the bridging I-links. While in the idealized model, the peak of the von Mises stress of the stent was $418.3 \mathrm{MPa}$, and the exact position of this peak changed although it was still located at the bend of the crown. The stress distribution in the stent of the idealized model was symmetric. The maximum von Mises stress was 405.7 $\mathrm{MPa}$ in the realistic model when the systolic pressure was $120 \mathrm{mmHg}$, as shown in Fig. 6(b). When the diastolic pressure was $80 \mathrm{mmHg}$, the position of the maximum von Mises stress changed, and the value was $407.9 \mathrm{MPa}$, as shown in Fig. 6(c). The maximum von Mises stress occurred in the expansion step. In the idealized model, the maximum von Mises stress was $412.3 \mathrm{MPa}$ (Fig. 6(b)) and $413.167 \mathrm{MPa}$ (Fig. 6(c)).

By comparing the stress contours in different stages, the large deformation in the expansion stage proved a potential factor in affecting fracture of the stent. In addition, the von Mises stress in the stent of the idealized model was higher than that of the realistic model. Thus, the realistic geometry parameters were proved to be a relevant mechanical occurrence, including a realistic plaque which might affect the risk of the static failure of metallic stents.

It was evident that the highest stresses were observed near the connectors between the stent struts. This conclusion was similar to the findings by Zhang et al. [2014] and Gijsen et al. [2008]. In addition, higher stresses were observed in the stent struts close to the calcified plaque region, which was in agreement with the finding of Schievano et al. 2010].

\subsection{Influence of a realistic geometry: fatigue life analysis}

The FDA recommends that Goodman analysis should be used to determine the fatigue resistance of the stent to physiologic loading. Goodman fatigue life analysis has been widely used for assessing the device fatigue resistance and providing an 


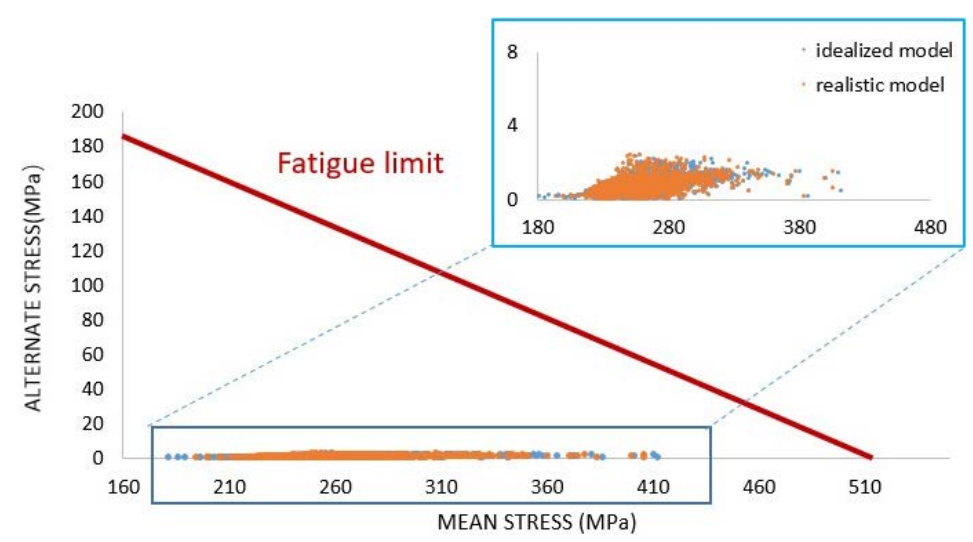

Fig. 7. (Color online) Goodman diagrams for fatigue analysis using the idealized model (blue) and using the realistic model (orange).

indication of device chronic durability. The endurance limit for zero mean stress of the 304SS material was assumed to be $186 \mathrm{MPa}$ while the ultimate stress was equal to $520 \mathrm{MPa}$ Li et al. (2013); Jayaprakash et al. (2013)]. We followed these data to establish the Goodman diagram (Fig. 7).

Values of the effective mean and alternative stresses were calculated at the nodes of the stent with a high von Mises stress. The red line in Fig. 7 referred to the limit of the stent material. In theory, the closer the stress point was to the fatigue limit, the more dangerous was the structure. As shown in Fig. 7 all the points fell below the limit line of the material, which meant they were in safety. Compared with the realistic model, the nodes in the idealized model were more scattered in the diagram and some nodes were closer to the fatigue limit line.

Based on the mechanical analysis, the most dangerous 10 nodes in the realistic model were selected, and the calculated data of the idealized model was compared.

Table 2. The fatigue performance parameters of the most dangerous points.

\begin{tabular}{|c|c|c|c|c|c|c|}
\hline \multirow[b]{2}{*}{ Number } & \multicolumn{2}{|c|}{ Predicted number of cycles to failure } & \multicolumn{2}{|c|}{ Fatigue life (a) } & \multicolumn{2}{|c|}{ FSF } \\
\hline & $\begin{array}{l}\text { Using the } \\
\text { realistic } \\
\text { model }\end{array}$ & $\begin{array}{c}\text { Using the } \\
\text { idealized } \\
\text { model }\end{array}$ & $\begin{array}{l}\text { Using the } \\
\text { realistic } \\
\text { model }\end{array}$ & $\begin{array}{l}\text { Using the } \\
\text { idealized } \\
\text { model }\end{array}$ & $\begin{array}{c}\text { Using the } \\
\text { realistic } \\
\text { model }\end{array}$ & $\begin{array}{c}\text { Using the } \\
\text { idealized } \\
\text { model }\end{array}$ \\
\hline 1 & $5.57 \mathrm{E}+08$ & $4.80 \mathrm{E}+08$ & 14.66 & 12.63 & 1.35 & 1.34 \\
\hline 2 & $7.57 \mathrm{E}+08$ & $6.39 \mathrm{E}+08$ & 19.92 & 16.82 & 1.36 & 1.35 \\
\hline 3 & $8.91 \mathrm{E}+08$ & $9.09 \mathrm{E}+08$ & 23.44 & 23.92 & 1.37 & 1.38 \\
\hline 4 & $1.03 \mathrm{E}+09$ & $1.00 \mathrm{E}+09$ & 27.05 & 26.32 & 1.39 & 1.39 \\
\hline 5 & $1.08 \mathrm{E}+09$ & $9.56 \mathrm{E}+08$ & 28.34 & 25.15 & 1.39 & 1.38 \\
\hline 6 & $1.81 \mathrm{E}+09$ & $1.45 \mathrm{E}+09$ & 47.74 & 38.24 & 1.43 & 1.41 \\
\hline 7 & $2.90 \mathrm{E}+09$ & $2.39 \mathrm{E}+09$ & 76.29 & 63.03 & 1.46 & 1.45 \\
\hline 8 & $3.00 \mathrm{E}+09$ & $2.78 \mathrm{E}+09$ & 78.99 & 73.25 & 1.47 & 1.46 \\
\hline 9 & $3.26 \mathrm{E}+09$ & $2.02 \mathrm{E}+09$ & 85.72 & 53.03 & 1.47 & 1.43 \\
\hline 10 & $3.29 \mathrm{E}+09$ & $2.25 \mathrm{E}+09$ & 86.63 & 59.19 & 1.47 & 1.44 \\
\hline
\end{tabular}


The data of the most dangerous points predicted by these two models was shown in Table 2.

According to the ascending order of fatigue life rate, the predicted cycles to failure of the most dangerous point were $5.57 \times 10^{8}(14.7$ years equivalent $)$ in the realistic model, while that in the idealized model were $4.80 \times 10^{8}(12.7$ years equivalent). The relative error ratio was about $13.8 \%$ on the basis of the former one. We also calculated the FSF, which was defined as the ratio of the strain amplitude against the modified endurance limit, and a FSF less than 1.0 indicated the stent fatigue failure. The trend of FSF was similar to that of the fatigue cycle, while the fatigue cycle quantified the proximity of the mean strain and strain amplitude at the given integration points to the Goodman failure line. In addition, the prediction of FSF in the realistic model was higher (Table 2). The difference between these two different models indicated that it was necessary to analyze mechanical parameters and fatigue life by using a realistic model.

\section{Conclusion}

In this study, the interaction of the stents, the plaque and the vessel wall in a realistic model and an idealized model were simulated. The von Mises stress in the stent was similar in these two models. The maximum stress during the expansion and recoiling stages was always located at the inner bend of the crowns. However, the von Mises stresses in the plaque and in the vessel wall varied largely in different models. Based on the mechanical analysis, the effects of cyclic loading on stent fatigue rupture were investigated. To evaluate the fatigue performance accurately, we recommend using the realistic coronary stenosis model. This study also provides an approach to select and position a stent to minimize the risk of fatigue fracture. In fact, this is a general approach and not limited to 304 stainless steel, and further applications should focus on other metallic materials.

This study can be improved by the presence of some limitations. From a modeling point of view, the absence of the balloon had an influence during the inflating process, and the influence of residual stress in the process of crimping was ignored. We also found that the effects of different properties for vessel and plaque materials on the stents' mechanical fatigue should not be ignored. Future research is devoted to removing this assumption to have a more realistic description of the stent fatigue prediction. In addition, the influence of the cardiac wall movement on the stent fracture should be taken into account in future works. In this paper, material data cited as the strain-based Goodman fatigue life analysis were from several references. We need more material tests to get the material data to acquire results that are more accurate.

The information in the present study may be helpful in predicting the stent strut fracture and assessing the fatigue life for a coronary stent. This study may eventually help designers to optimize the stent geometry structure and help physicians with their diagnosis and intervention surgical decision-making process. 


\section{Acknowledgments}

This work was supported by Major Project of Science and Technology of Beijing Municipal Education Commission and Type B Project of Beijing Natural Science Foundation (KZ201710005007).

\section{References}

Almangour, B., Mongrain, R. and Yue, S., [2013] "Coronary stents fracture: An engineering approach (review)," Materials Sciences 85 Applications 4(10), 606-621.

Auricchio, F., Conti, M., De, B. M. et al. [2011] "Carotid artery stenting simulation: From patient-specific images to finite element analysis," Medical Engineering $\& 3$ Physics 33(3), 281.

Auricchio F, Constantinescu, A., Conti, M. et al. [2015] "Fatigue of Metallic Stents: From Clinical Evidence to Computational Analysis," Annals of Biomedical Engineering 44(2), 1-15.

Bessias, N., Sfyroeras, G., Moulakakis, K. G. et al. [2005] "Renal artery thrombosis caused by stent fracture in a single kidney patient," Journl of Endovascular Therapy 12(4), $516-520$.

Chua, S. N. D., Macdonald, B. J. and Hashmi, M. S. J. [2004] "Finite element simulation of slotted tube (stent) with the presence of plaque and artery by balloon expansion," Journal of Materials Processing Technology 6, 1772-1779.

Dordoni, E., Meoli, A., Wu, W. et al. [2014] "Fatigue behaviour of Nitinol peripheral stents: The role of plaque shape studied with computational structural analyses," Medical Engineering \& Physics 36, 842-9.

Gijsen, F. J., Migliavacca, F., Schievano, S. et al. [2008] "Simulation of stent deployment in a realistic human coronary artery," Biomedical Engineering Online 7, 23-23.

Hsiao, H. M., Chiu, Y. H., Lee, K. H. et al. [2012] "Computational modeling of effects of intravascular stent design on key mechanical and hemodynamic behavior," ComputerAided Design 44, 757-765.

Hsiao, H. M., Wu, L. W., Yin, M. T. et al. [2014] "Quintupling fatigue resistance of intravascular stents via a simple design concept," Computational Materials Science 86, $57-63$.

Jayaprakash, M., Anchalee, S., Otsuka et al. [2013] "Tsr-csr diagram for 304 stainless steel." International Journal of Fatigue 54(9), 99-105.

Lewitton, S. and Babaev, A. [2008] "Superficial Femoral Artery Stent Fracture that Led to Perforation, Hematoma and Deep Venous Thrombosis," Journal of Invasive Cardiology 20, 479-81.

Li, H. X., Qiu, T., Zhu, B., Wu, J. et al. [2013] "Design optimization of coronary stent based on finite element models," The Scientific World Journal 5, 630243(1-10).

Lloydjones, D., Adams, R. J., Brown, T. M. et al. [2010] "Heart disease and stroke statistics - 2010 update a report from the american heart association," Circulation 121(7), e46.

Marrey, R. V., Burgermeister, R., Grishaber, R. B. et al. [2006] "Fatigue and life prediction for cobalt-chromium stents: A fracture mechanics analysis," Biomaterials 27, 19882000.

Morlacchi, S., Pennati, G., Petrini, L., et al. [2014] "Influence of plaque calcifications on coronary stent fracture: A numerical fatigue life analysis including cardiac wall movement," Journal of Biomechanics 47(4), 899-907. 
Pericevic, I., Lally, C., Toner, D., et al. [2009] "The influence of plaque composition on underlying arterial wall stress during stent expansion: The case for lesion-specific stents," Medical Engineering 83 Physics 31(4), 428-433.

Petrini, L., Trotta, A., Dordoni, E. et al. [2016] "A Computational Approach for the Prediction of Fatigue Behaviour in Peripheral Stents: Application to a Clinical Case," Annals of Biomedical Engineering 44.2, 536-547.

Qiao, A. and Zhang, Z. [2014] "Numerical simulation of vertebral artery stenosis treated with different stents," J Biomech Eng 136(4), 1274-1283.

Ragkousis, G. E., Curzen, N., Bressloff, N. W. [2014] "Simulation of longitudinal stent deformation in a patient-specific coronary artery," Medical Engineering 83 Physics 36, 467-476.

Scheinert, D., Scheinert, S., Sax, J. et al. [2005] Prevalence and clinical impact of stent fractures after femoropopliteal stenting," Journal of the American College of Cardiology 45. 2, 312-315.

Schievano, S., Taylor, A. M., Capelli, C. et al. [2010] "Patient specific finite element analysis results in more accurate prediction of stent fractures: Application to percutaneous pulmonary valve implantation," Journal of Biomechanics 43, 687-693.

Yang, Z., Zhang, H. P. and Marder, M. [2008] "Dynamics of static friction between steel and silicon," Proceedings of the National Academy of Sciences of the United States of America 105, 13264-13268. 